\title{
Mechanical Properties of Soft Biological Membranes for Organ-on-a- Chip Assessed by Bulge Test and AFM
}

\author{
Pauline Zamprogno, ${ }^{\dagger}$ Giuditta Thoma, ${ }^{\dagger}$ Veronika Cencen, Dario Ferrari, Barbara Putz, Johann Michler, \\ Georg E. Fantner, and Olivier T. Guenat*
}

Cite This: https://dx.doi.org/10.1021/acsbiomaterials.0c00515

Read Online

ACCESS | 岁 Metrics \& More | 回 Article Recommendations

SI Supporting Information

ABSTRACT: Advanced in vitro models called "organ-on-a-chip" can mimic the specific cellular environment found in various tissues. Many of these models include a thin, sometimes flexible, membrane aimed at mimicking the extracellular matrix (ECM) scaffold of in vivo barriers. These membranes are often made of polydimethylsiloxane (PDMS), a silicone rubber that poorly mimics the chemical and physical properties of the basal membrane. However, the ECM and its mechanical properties play a key role in the homeostasis of a tissue. Here, we report about biological membranes with a composition and mechanical properties similar to those found in vivo. Two types of collagenelastin (CE) membranes were produced: vitrified and nonvitrified

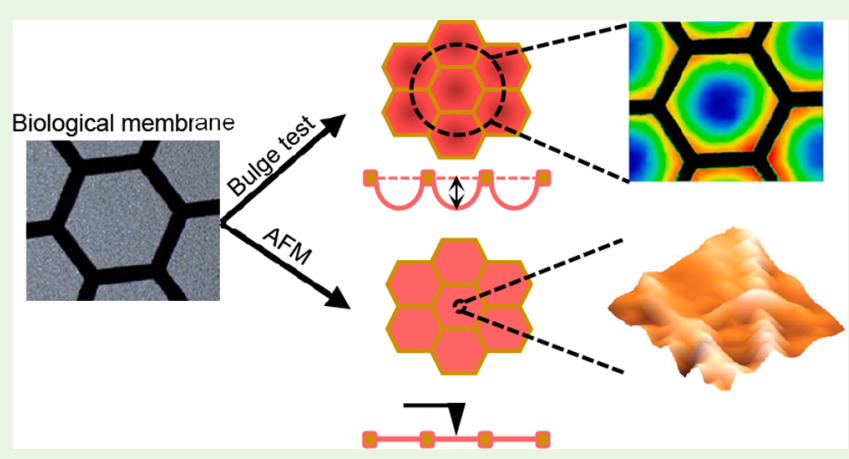
(called "hydrogel membrane"). Their mechanical properties were characterized using the bulge test method. The results were compared using atomic force microscopy (AFM), a standard technique used to evaluate the Young's modulus of soft materials at the nanoscale. Our results show that $\mathrm{CE}$ membranes with stiffnesses ranging from several hundred of $\mathrm{kPa}$ down to $1 \mathrm{kPa}$ can be produced by tuning the CE ratio, the production mode (vitrified or not), and/or certain parameters such as temperature. The Young's modulus can easily be determined using the bulge test. This method is a robust and reproducible to determine membrane stiffness, even for soft membranes, which are more difficult to assess by AFM. Assessment of the impact of substrate stiffness on the spread of human fibroblasts on these surfaces showed that cell spread is lower on softer surfaces than on stiffer surfaces.

KEYWORDS: ECM membrane, organ-on-a-chip, bulge test, atomic force microscopy, Young's modulus

\section{INTRODUCTION}

In vivo, cells reside in a complex microenvironment that is constantly exposed to endogenous and exogenous forces. These forces are transferred to the cytoskeleton via integrins and the extracellular matrix (ECM). The mechanical properties of the ECM strongly influence cellular morphology, proliferation, and differentiation. ${ }^{1-3}$ In the human body, the mechanical properties of different tissues vary according to composition and location. Most organs, such as the lungs, brain, or kidneys, are compliant and have a stiffness lower than $15 \mathrm{kPa}^{4}$ Therefore, soft materials are required to reproduce the physiological microenvironment of cells in in vitro systems.

Organ-on-a-chip (OOC) technology often uses polydimethylsiloxane (PDMS) membranes to reproduce the in vivo barrier. Because of the mechanical properties of this material, OOC membranes can stretch to mimic breathing ${ }^{5,6}$ or heartbeat $^{7,8}$ motion. PDMS has several advantages: it can easily be microstructured using soft lithography and is permeable to oxygen, optically transparent, biocompatible, and cost-effective. ${ }^{9}$ However, it also has several limitations, of which an important one is a high propensity for ad- and absorption of small molecules such as drugs. ${ }^{10,11}$ In addition, it is an artificial substance and thus does not mimic the chemical composition of the ECM. To overcome these limitations, researchers have recently developed collagen-based membranes integrated in OOCs. ${ }^{12-16}$ The biological and structural properties of the membrane can be tuned by changing the composition and concentration of the membrane materials to mimic the ECM properties of a specific tissue. ${ }^{17}$ Collagen is biocompatible, biodegradable, and can easily be structured into a scaffold. Collagen-based membranes can mimic the chemical composition and structure of the in vivo ECM, and unlike PDMS, they do not show high ab- and adsorption capacity. ${ }^{13}$ Combinations of collagen and elastin are used to develop living tissues such as cardiovascular, skin, liver, and musculoskeletal tissues. $^{18}$

Special Issue: Beyond PDMS and Membranes: New

Materials for Organ-on-a-Chip Devices

Received: April 9, 2020

Accepted: February 12, 2021 
Atomic force microscopy (AFM)-based testing is one of the most commonly used methods to characterize biological membranes. ${ }^{19-21}$ However, although imaging and mechanical sensing by AFM appear straightforward, several intricacies complicate the acquisition of quantitative data, making the method time- and labor-intensive. ${ }^{19}$ Furthermore, AFM provides local stiffness values that do not necessarily reflect the average stiffness of the material. Sheng et al. ${ }^{22}$ recently reported the use of the bulge test, a method developed for rigid thin films, ${ }^{23}$ for the mechanical characterization of soft membranes. In this nondestructive method, the mechanical properties of the analyzed material are determined by measuring the relationship between the deflection of freestanding membranes and the applied pressures. ${ }^{24-26}$

In this study, we describe two types of biological membranes made of collagen and elastin (CE) supported by a gold mesh, namely, vitrified and nonvitrified (called "hydrogel") CE membranes. We report the mechanical characterization of these membranes using AFM and the bulge test method. The mechanical properties of the membranes were assessed as a function of several parameters, such as the concentration of collagen and the fabrication temperature. The effect of the membrane's stiffness on biological systems was assessed by evaluating the morphological behavior of lung fibroblasts cultured on the membranes.

\section{MATERIALS AND METHODS}

Production of the CE Membrane and Chip Fabrication. The production of the vitrified $\mathrm{CE}$ membrane and the chip fabrication procedure were described earlier. ${ }^{13}$ In brief, the biological membranes were made of rat-tail collagen type I, high concentration (Corning, New York, NY, USA), and bovine neck elastin lyophilized powder (Sigma-Aldrich, Buchs, Switzerland). The two molecules were mixed at a ratio of $1: 1$ to a final concentration of $3.5 \mathrm{mg} / \mathrm{mL}$ in a $\mathrm{pH} 7.4$ buffer. An $18 \mu \mathrm{m}$ thin gold mesh (Plano GmbH, Wetzlar, Germany) with hexagonal pores of $500 \mu \mathrm{m}$ was used as a scaffold to create the biological membrane. The CE solution was pipetted directly over the gold mesh. After pipetting, the chip was immediately incubated at 37 ${ }^{\circ} \mathrm{C}, 100 \%$ humidity, and $5 \% \mathrm{CO}_{2}$ for $1 \mathrm{~h}$ to allow gelation of the membrane. For membranes produced at $4{ }^{\circ} \mathrm{C}$, the membrane was placed in the refrigerator overnight. For the hydrogel CE membrane, the membrane was immediately hydrated using cell culture medium. For the vitrified CE membrane, the membrane was dried at room temperature for $48 \mathrm{~h}$ before rehydration using cell culture medium for $2 \mathrm{~h}$ at $37^{\circ} \mathrm{C}$. The membrane was sandwiched between a PDMS top layer and a polycarbonate bottom with double-sided tape (Figure S1). Prior to the membrane fabrication, the microfluidic device was sterilized with ozone (CoolCLAVE, Genlantis) and exposed to UV light for $45 \mathrm{~min}$. A $10 \mu \mathrm{m}$ thin PDMS Sylgard 184 membrane produced as described previously ${ }^{13}$ was used as a reference material.

Bulge Test. In the bulge test, uniform air pressure is applied to one side of a freestanding membrane, and the resulting deflection, called the bulge height, is measured. For a membrane with a circular shape, the pressure $(P)$ is expressed as a function of the bulge height (h) as follows:

$$
P=\frac{4 \sigma_{0} t}{a^{2}} h+\frac{8 E t}{3 a^{4}(1-\nu)} h^{3}
$$

where $E$ and $\sigma_{0}$ are the Young's modulus and the residual stress of the membrane, respectively; $t$ is the thickness; $a$ is the radius; and $\nu$ is the Poisson ratio. ${ }^{23}$ Equation 1 was applied to the hexagonal gold mesh, and the radius was obtained by averaging the diameters of the circles inscribing and circumscribing the hexagons. The membrane was deflected using a homemade electropneumatic system that generates a negative pressure that can be tuned up to $30 \mathrm{kPa}$. The deflection was visualized with a 3D optical profiler (S neox, Sensofar, Spain), whereas deflection measurements were obtained using an AxioPlan2 Zeiss microscope equipped with an incubator. The deflection values were obtained by measuring the difference in height between the stretched and unstretched membrane using focus variations on the membrane surface at the center of a hexagon. The deflection of the gold mesh itself was subtracted from the total deflection value. The deflection values of the PDMS, the vitrified CE membrane, and the hydrogel $\mathrm{CE}$ membrane were determined using the following pressure ranges: $0-4 \mathrm{kPa}$ for the PDMS and CE-vitrified membranes, and 0-2 $\mathrm{kPa}$ for the CE-hydrogel membrane, with increments of 0.5 and 0.3 $\mathrm{kPa}$, respectively. At least three membranes were tested per condition. The experimental pressure-deflection curves were analyzed using MATLAB software and fitted to eq 1, from which the Young's modulus was extrapolated. The membrane was in contact with the cell culture medium in the basolateral compartment during all deflection measurements.

AFM. AFM stiffness measurements were performed in-liquid (cell culture medium on the apical side of the membrane) using a customized Dimension Icon AFM head (Bruker Nano Surface, Santa Barbara, California, US) mounted on an inverted optical microscope. ${ }^{27}$ For the measurement, a $70 \mu \mathrm{m}$ thick PDMS layer was bonded on a glass slide and four layers of CE-vitrified membrane (pipetted at $3.2 \mu \mathrm{L} / \mathrm{mm}^{2}$ ) were directly produced on this substrate. Force-volume images were acquired within a square of $30 \mu \mathrm{m} \times 30$ $\mu \mathrm{m}$ at the center of the membrane. A minimum of 256 points were measured per membrane. The bead cantilevers CP-qp-CONT-PS (sQube) with a $3.6 \mu \mathrm{m}$ radius bead were used for indenting the membrane. Data analysis was performed using Nanoscope Analysis software (Bruker), and the Young's modulus was calculated using the Hertzian model.

Thickness Measurement. The thickness of the vitrified CE membrane was measured as described previously. ${ }^{13}$ In brief, the membrane was cut at the center and imaged using the reflective light microscopy mode of the AxioPlan2 Zeiss microscope. The thickness was measured using Axiovision software. For the hydrogel membrane, the thickness was measured with the same microscope as a Z-stack between the bottom and the top of the gel. To help identify the membrane surface and thus increase the accuracy of the measurement, graphite powder (obtained by scratching a pencil tip with a scalpel) was spread on the hydrogel $\mathrm{CE}$ membrane. Four samples were measured for each condition.

Cell Culture. The human fetal lung fibroblast cell line HFL-1 was obtained from ATCC (CCL-153) and cultured in F-12K medium (Gibco) supplemented with 10\% FBS (Sigma) and 1\% p/s. Cells were used between passages 25 and 27, and seeded on chips at a density of $8 \times 10^{3}$ cells $/ \mathrm{cm}^{2}$. The PDMS membrane was coated overnight with a solution of collagen I (Sigma-Aldrich) and fibronectin (Corning). The membrane was washed and then dried under UV for $45 \mathrm{~min}$. After 24 $\mathrm{h}$, cells were fixed and analyzed by immunofluorescence. All cell manipulations were performed in a sterile flow hood, and cells were maintained at $37{ }^{\circ} \mathrm{C}, 100 \%$ humidity, and $5 \% \mathrm{CO}_{2}$.

Immunofluorescence. The chips were washed three times with PBS, fixed with $4 \%$ paraformaldehyde (Sigma-Aldrich) for $10 \mathrm{~min}$, and rinsed again three times with PBS. The cells were permeabilized with $0.1 \%$ Triton X-100 (Sigma-Aldrich) for $10 \mathrm{~min}$, washed three times with PBS, blocked for $45 \mathrm{~min}$ in a $2 \%$ BSA (Sigma-Aldrich) solution, and incubated in blocking solution containing Hoechst and actin for $1 \mathrm{~h}$. After the incubation, the chips were washed three times with PBS. All immunostaining steps were performed at room temperature. Images were acquired using a confocal microscope (CLSM, Zeiss LSM 710) and Leica DMI400 (Leica Microsystems, Buffalo Grove, IL, USA), and analyzed using Fiji software.

Cell Shape Analysis. To quantify the variation in cell morphology, we calculated the cell shape index (CSI), a dimensionless quantitative measure, using the following equation:

$$
\mathrm{CSI}=4 \pi \frac{S}{p^{2}}
$$




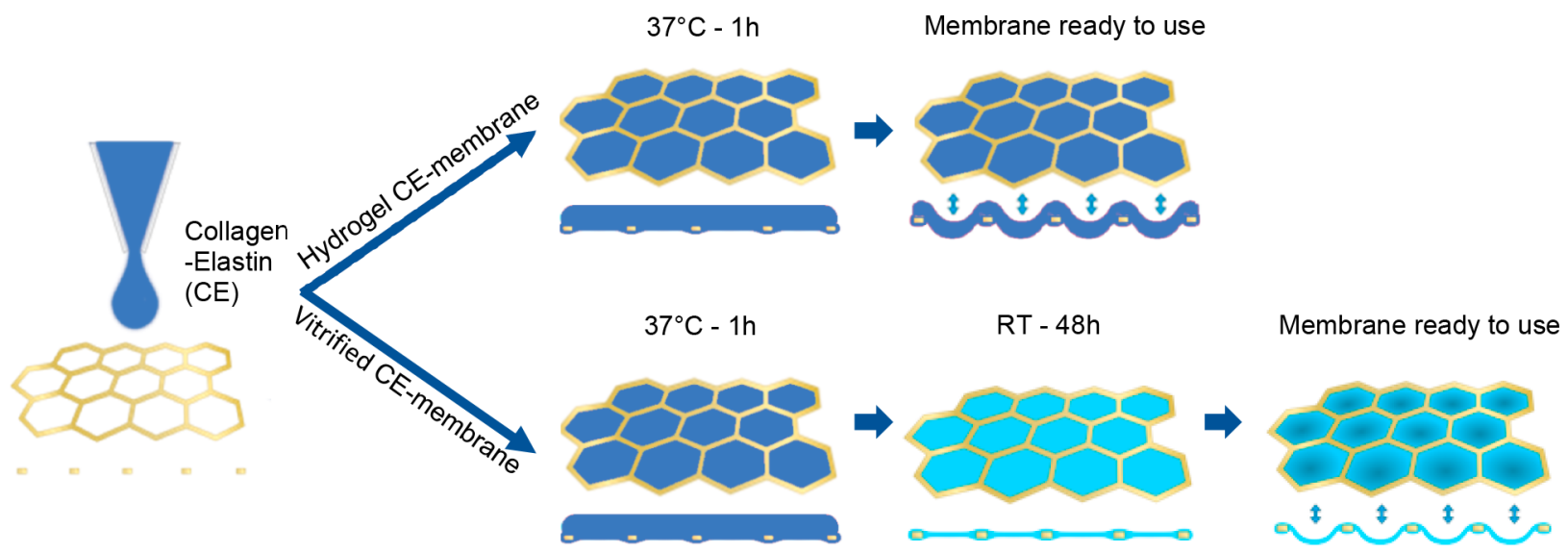

Figure 1. Fabrication procedure of the hydrogel and vitrified CE membranes. Modified with permission from ref 13. CC-BY. A drop of collagen and elastin is pipetted on a thin gold grid, which results in the spreading of the solution by surface tension force. Two membrane types were investigated. After gelation, the hydrogel membranes (top) were left in cell culture medium, whereas the vitrified membranes (bottom) were dried and rehydrated prior to characterization.

(A)

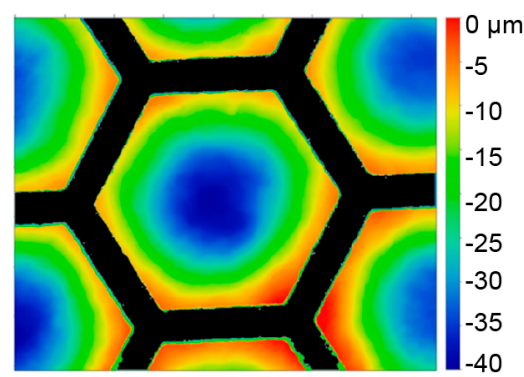

(C)

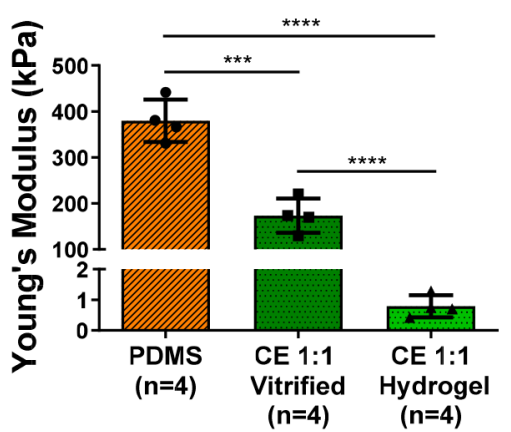

(B)
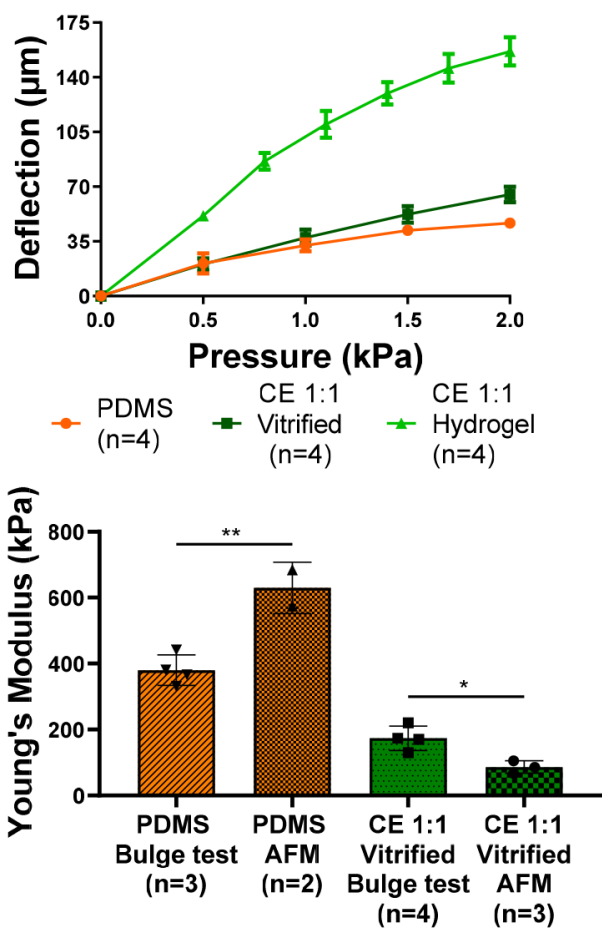

Figure 2. Mechanical characterization of biological membranes. (A) Visualization of the vitrified CE membrane deflection under a negative pressure of $0.5 \mathrm{kPa}$ with an optical profiler. (B) Deflection of hydrogel and vitrified CE membranes as a function of applied pressure. Tenmicrometer-thin PDMS membrane was used as a reference. (C) Young's modulus of membranes extrapolated from the pressure-deflection curves. (D) Comparison of Young's modulus obtained via AFM and bulge test.

where $S$ is the surface of the cell and $p$ is the perimeter. The surface and the perimeter were measured using Fiji software.

Statistics. Data are presented as the mean \pm standard deviation (SD). The significance of differences was assessed using the two-tailed unpaired Student's $t$ test. Statistical significance was defined as follows: $* p<0.05, * * p<0.01, * * * p<0.001$, and $* * * * p<0.0001$. Statistical analysis was performed using GraphPad Prism 8 software.

\section{RESULTS}

Formation of Vitrified and Hydrogel CE Membranes. The biological membranes were generated using a simple process based on surface tension (Figure 1). A drop of CE solution was pipetted onto a thin gold mesh. Two fabrication procedures were evaluated to produce a vitrified membrane or a hydrogel membrane. First, a gelation step was used to promote cross-linking of the CE molecules. This step created a hydrogel CE membrane that could be used immediately as support for cells. To generate the vitrified CE membrane, we dried the CE solution at room temperature for 2 days. This evaporation process created a vitrified CE membrane that was 12-fold thinner than the hydrogel CE membrane (Figure S2). The thickness of the vitrified CE membrane was $8.2 \pm 2.0 \mu \mathrm{m}$, whereas that of the hydrogel CE membrane was $100.1 \pm 28.8$ $\mu \mathrm{m}$ when $1.6 \mu \mathrm{L} / \mathrm{mm}^{2}$ of the CE solution was pipetted onto 
(A)

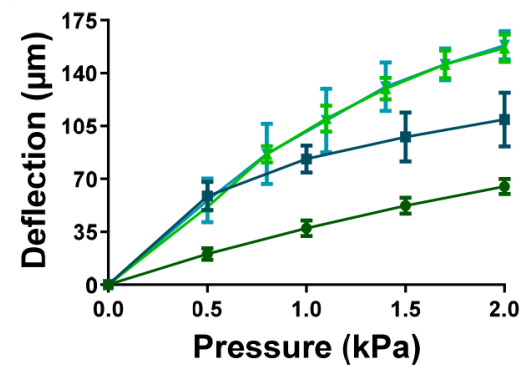

(B)

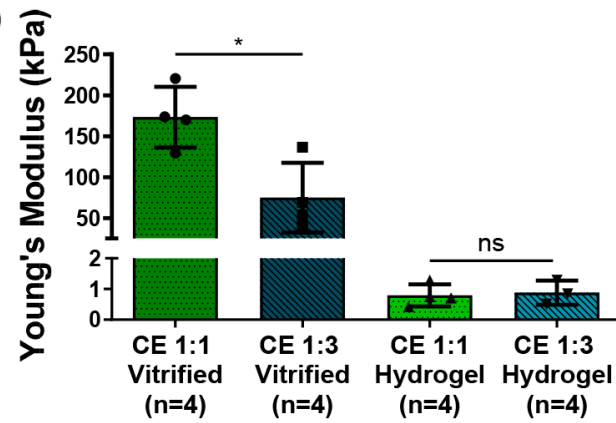

Figure 3. Mechanical characterization of biological membranes as a function of protein ratio. (A) Pressure-deflection curves of vitrified and hydrogel CE membranes with varying ratios of collagen to elastin. (B) Young's modulus extracted from pressure-deflection curves as a function of chemical composition.

(A)

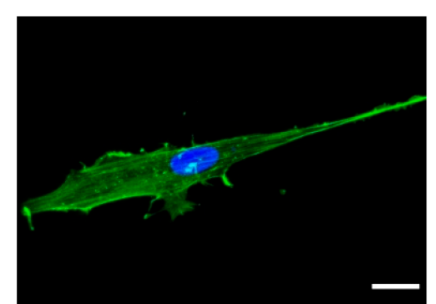

PDMS

$S=2428 \mu \mathrm{m}^{2}$

$\mathrm{CSI}=0,1$

(B)

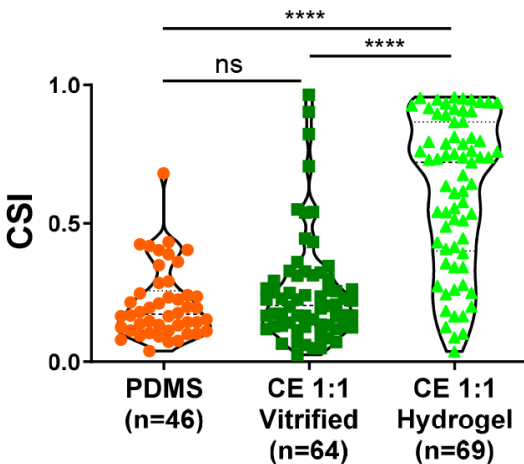

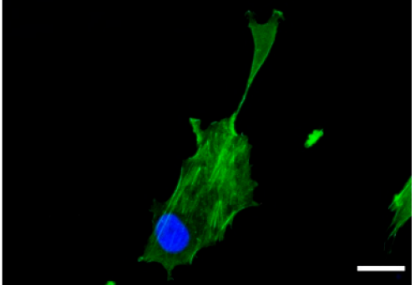

CE 1:1 Vitrified $S=1899 \mu \mathrm{m}^{2}$ $\mathrm{CSI}=0,29$

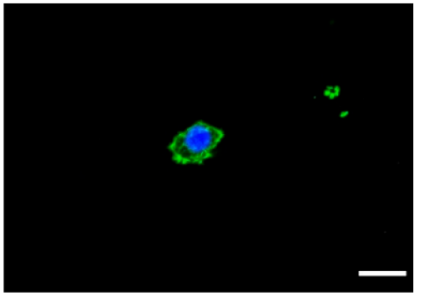

CE 1:1 Hydrogel

$S=280,9 \mu \mathrm{m}^{2}$

$\mathrm{CSI}=0,81$

(C)

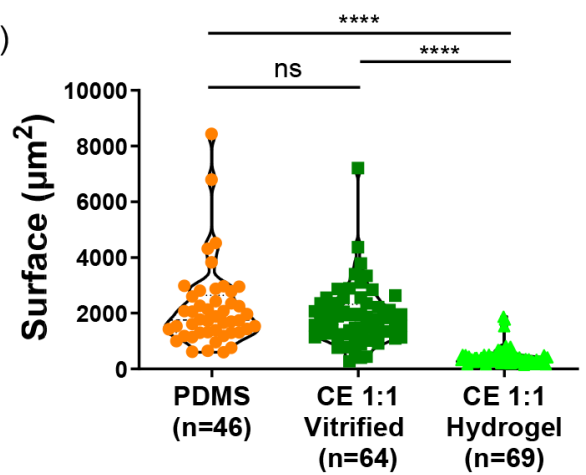

Figure 4. Effect of the substrate stiffness on cell spreading. (A) HFL-1 on PDMS, CE 1:1 vitrified, and CE 1:1 hydrogel membrane. Scale bar: 20 $\mu \mathrm{m}$. (B) Cell shape index (CSI) and (C) cell surface of HFL-1 on PDMS, CE 1:1 vitrified, and CE 1:1 hydrogel membranes.

the mesh. The vitrified CE membranes were rehydrated in cell culture medium for $2 \mathrm{~h}$ before characterization.

Mechanical Characterization of Thin Membranes: AFM vs Bulge Test. The two types of developed biological membrane were flexible and could be deflected when exposed to negative pressure (Figure 2A and Figure S3). The fabrication process affected the stretchability of the biological membranes (Figure 2B). At $0.5 \mathrm{kPa}$, the deflection was $51.4 \pm$ $3.1 \mu \mathrm{m}$ for the hydrogel CE membrane and $20.4 \pm 3.8 \mu \mathrm{m}$ for the vitrified CE membrane. Deflection was 2- to 3-fold higher for biological membranes than for the $10 \mu \mathrm{m}$ PDMS membrane used as reference (Figure 2B). At $2 \mathrm{kPa}$, the deflection was $156.6 \pm 9.2 \mu \mathrm{m}$ for the hydrogel CE membrane, $65.0 \pm 5.0 \mu \mathrm{m}$ for the vitrified CE membrane, and $46.7 \pm 2.0$ $\mu \mathrm{m}$ for the PDMS membrane. Young's modulus was extrapolated from the relation between the deflection of the membrane and the pressure applied (Figure S4). The Young's modulus of the PDMS membrane $\left(E_{\mathrm{PDMS}}=380 \pm 46 \mathrm{kPa}\right)$ evaluated using the bulge test method was approximately 2fold higher than that of the vitrified $\mathrm{CE}$ membrane $\left(E_{\mathrm{CE}, \mathrm{v} .}=\right.$ $173 \pm 37 \mathrm{kPa}$ ), and approximately 200-fold higher than that of the hydrogel CE membrane $\left(E_{\mathrm{CE}, \mathrm{h} .}=0.79 \pm 0.36 \mathrm{kPa}\right)$ (Figure 2C). A slight, but not significant, increase in stiffness was observed after storing the vitrified $\mathrm{CE}$ membrane at room temperature for 14 days (Figure S5). However, the membrane stiffness did not change after immersion in cell culture medium for 2 weeks at $37{ }^{\circ} \mathrm{C}$ (Figure S6). The Young's moduli were also determined by AFM via the acquisition of force volume images (Figure S7). Layers of the respective materials were produced on a glass slide to avoid the geometric effects one would have when measuring directly the suspended membranes. The stiffness for the PDMS was of $630 \pm 78 \mathrm{kPa}$, and 
$86 \pm 19 \mathrm{kPa}$ for the vitrified CE layer. The variation of the AFM data is shown in Figure S8. The thicknesses of the layers were chosen so that the stiffness of the glass would not affect the measurement. The stiffness of the hydrogel CE membrane was only evaluated with the bulge test because of the difficulty in identifying the contact point between the cantilever tip and the surface of the hydrogel using AFM.

Impact of CE Ratio and Gelation Temperature on the Mechanical Properties of the Membranes. The mechanical properties of the vitrified CE membranes varied according to the composition of the solution pipetted onto the gold mesh. At lower collagen concentrations, the vitrified $\mathrm{CE}$ membrane was softer, which resulted in larger deflection values. For example, at $1 \mathrm{kPa}$, the deflection was $83.2 \pm 8.9 \mu \mathrm{m}$ at a $1: 3$ ratio and $37.5 \pm 5.2 \mu \mathrm{m}$ at a $1: 1$ ratio of collagen to elastin (Figure 3A). The resulting Young's modulus for the vitrified $\mathrm{CE}$ membrane $1: 3\left(E_{\mathrm{CE}, \mathrm{v} 1: 3}=75 \pm 43 \mathrm{kPa}\right)$ was approximately one-half of that for the $1: 1$ membrane $\left(E_{\mathrm{CE}, \mathrm{v}} 1: 1\right.$ $=173 \pm 37 \mathrm{kPa})($ Figure $3 \mathrm{~B})$. However, the protein ratio $(1: 1$ or $1: 3$ ) had no significant effect on the mechanical properties of the hydrogel CE membranes. Another factor affecting the stiffness of CE membranes is the gelation temperature. The Young's modulus of the hydrogel CE membrane produced at $37{ }^{\circ} \mathrm{C}\left(E_{\mathrm{CE}, \mathrm{h} \_37^{\circ} \mathrm{C}}=0.79 \pm 0.36 \mathrm{kPa}\right)$ was approximately 2 -fold lower than that of the membrane produced at $4{ }^{\circ} \mathrm{C}\left(E_{\mathrm{CE}, \mathrm{h} \_}{ }^{\circ} \mathrm{C}\right.$ $=2.0 \pm 0.54 \mathrm{kPa}$ ) (Figure S9).

Impact of Stiffness on Cell Spreading. To determine the effect of substrate stiffness on cell spreading, human lung fibroblasts were cultured on PDMS, vitrified CE, and hydrogel CE membranes. As shown in Figure 4A, the substrate type directly affected the shape and the cell surface characteristics. The fibroblasts on the hydrogel CE membrane have a circular shape characterized by a CSI of $0.62 \pm 0.27$, whereas on PDMS and on the vitrified CE membrane, the cells are elongated $\left(\mathrm{CSI}_{\mathrm{PDMS}}=0.21 \pm 0.13\right.$ and $\left.\mathrm{CSI}_{\mathrm{V} . \mathrm{CE}}=0.25 \pm 0.2\right)$ (Figure 4B). Additionally, the surface area of fibroblasts grown on PDMS and vitrified CE membranes was similar (2153 \pm $1479 \mu \mathrm{m}^{2}$ on PDMS and $1883 \pm 1041 \mu \mathrm{m}^{2}$ on the vitrified CE membrane), whereas that of cells grown on the hydrogel CE membrane was approximately 4-fold smaller $\left(517 \pm 333 \mu \mathrm{m}^{2}\right)$ (Figure 4C). These data indicate that the stiffness of the surrounding ECM affects cell morphology.

\section{DISCUSSION}

The structure, stiffness, elasticity, and composition of the ECM are important for the maintenance of tissue homeostasis in health and disease. ${ }^{28,29}$ Therefore, reproducing ECM characteristics in advanced in vitro models is critical. In OOC devices, thin and flexible membranes made of PDMS (Sylgard 184) mimic the basal membrane and act as a substrate for cells. ${ }^{5,6}$ However, despite several advantages for cell culture, the composition and mechanical properties of PDMS differ from those of soft tissues. To achieve better simulation of physiological systems, researchers developed biological vitrified $^{12-15}$ and hydrogel ${ }^{30,31}$ membranes and integrated them into in vitro models. Collagen I has been used extensively in this context because of its capacity to form a stable gel rapidly at the physiological temperature. In this study, we report the mechanical characterization of biological membranes produced with or without vitrification. The simple fabrication process based on surface tension reported earlier ${ }^{13}$ was extended and used for hydrogel membranes enabling the reproduction of stiffnesses, with elastic moduli in the low kilopascal range, typically found in soft tissues. The membranes are composed of collagen I and elastin, two of the most abundant ECM proteins. ${ }^{28}$ The mechanical characterization of the membranes is carried out by AFM and compared with the bulge technique, which is reported here for the first time to the best of our knowledge.

The mechanical characterization of a cell-substrate, particularly the measurement of stiffness, is important because this parameter strongly affects cellular behavior. ${ }^{32}$ The AFM indentation technique is a widely used method to characterize the stiffness of soft biological materials at the nanoscale. However, it only provides a local value of the stiffness, whereas the global stiffness of a tissue-here of a membrane-is often of interest. In addition, the fine-tuning of the indentation parameters is sample-specific and time-consuming. ${ }^{33}$ In terms of instrumentation, the AFM method requires a specific setup for each biological material to be tested, particularly the cantilevers used. The bulge test is an alternative method for the determination of the average stiffness of freestanding membranes. ${ }^{22}$ In this study, we demonstrate that this nondestructive technique is a valuable tool to determine the Young's modulus of thin and soft membranes, in particular those with low stiffness (in the $\mathrm{kPa}$ range). The Young's modulus determined for the collagen-elastin hydrogel membrane $(0.79 \pm 0.36 \mathrm{kPa})$ is in the range of the values found in the literature for collagen based hydrogel (about 0.5-2.4 $\mathrm{kPa}^{30,34-36}$ of similar concentration (about $3 \mathrm{mg} / \mathrm{mL}$ )). Very few studies have estimated the Young's modulus of reswollen vitrified collagen-based membrane. Mondrinos et al. ${ }^{12}$ reported a value of $660 \mathrm{kPa}$ for a collagen vitrified membrane, which decreased to $429 \mathrm{kPa}$ when mixed with Matrigel. In the present case, elastin made it possible to increase the flexibility of the biological membrane and reduced its Young's modulus (173 \pm $37 \mathrm{kPa}$ ). Both methods, the bulge test and the AFM, have their own limitations and operating challenges. On one hand, Young's moduli obtained with the bulge test depend on the accuracy of the membrane thickness measurement, often difficult to determine for hydrogel membranes. The accurate estimation of the deflection amplitude is an additional challenge, due to the not well-defined membrane-liquid interface. On the other hand, the assessment of mechanical properties of thin, soft and suspended membrane is challenging with the AFM technique. In fact, the interaction of the tip with the membrane leads to local deformations that are linked to the mechanical properties of the material but also induces the membrane to deflect, creating a measurement artifact. Complex set-ups ${ }^{37,38}$ are needed to circumvent this problem. In the present case, thick layers of the respective materials were produced on glass slides and measured for their stiffness. The Young's moduli obtained using the bulge test were on the same order of magnitude than those determined by AFM.

The properties of collagen scaffolds, especially their mechanical and transport properties, depend on several parameters, such as the protein source and concentration, gelation $\mathrm{pH}$, temperature, and other parameters of the fabrication process. Each of these factors can dramatically affect the mechanical properties of membranes. ${ }^{39}$ In this study, we identified protein concentration as a key parameter affecting the Young's modulus of membranes. The flexibility of the vitrified CE membrane was directly dependent on the ratio between collagen and elastin, and an increase in elastin concentration over collagen led to larger deflections. However, decreasing the concentration of collagen could prevent gelation 
and the complete formation of hydrogel CE membranes. Another parameter affecting the mechanical properties of biological membranes is the gelation temperature. The selfassembly process occurs at a faster rate at higher temperatures, which can reduce the diameter of fibrils. ${ }^{40-42}$ In this study, lower gelation temperatures $\left(4{ }^{\circ} \mathrm{C}\right.$ vs $37{ }^{\circ} \mathrm{C}$ in the normal procedure) increased the gelation time and potentially the size of the fibers. That may explain the difference in Young's modulus for the hydrogel $\mathrm{CE}$ membrane obtained at $4{ }^{\circ} \mathrm{C}$ and at $37{ }^{\circ} \mathrm{C}$. These results suggest that membranes with a wide range of stiffness values (i.e., $0.8-200 \mathrm{kPa}$ ) can be generated by modifying the fabrication procedure and certain parameters such as protein concentration and gelation temperature, thus reproducing the stiffness of healthy tissues such as the brain $(0.5-1 \mathrm{kPa}),{ }^{43}$ lung $(1-5 \mathrm{kPa}),{ }^{44}$ and skin $(50 \mathrm{kPa}),{ }^{45}$ as well as fibrotic tissues $(20-100 \mathrm{kPa}){ }^{46}$

The mechanical properties of the surrounding substrate greatly influence cell morphology, ${ }^{47,48}$ proliferation, ${ }^{49}$ adhesion, ${ }^{50}$ migration, ${ }^{51}$ and differentiation. ${ }^{52,53}$ In particular, the stiffness of the ECM critically determines cell morphology and specifically cell spreading. We observed that fibroblasts cultured on stiff membranes (PDMS and CE-vitrified) have a significantly larger surface and lower CSI than those grown on soft materials (hydrogel CE membrane), which is in agreement with similar studies. ${ }^{2,54}$ However, the surface area did not differ between substrates exposed to $200 \mathrm{kPa}$ and those exposed to $400 \mathrm{kPa}$. Unlike the process for PDMS membranes, the biological membranes did not require any preliminary treatment for cell culture.

\section{CONCLUSION}

This study describes the mechanical characterization of new flexible biological membranes using the bulge test method compared with AFM. The results obtained with the nondestructive bulge technique were on the same order of magnitude as those obtained by AFM. The results showed that the bulge test is a robust and reproducible technique to determine the Young's modulus of thin biological suspended membranes. Stiffnesses as low as $1 \mathrm{kPa}$ could be assessed in an uncomplicated manner. The membranes presented in this study can be modified to reproduce the physiological or pathophysiological microenvironments of various tissues. In addition, these biological membranes fabricated out of natural materials are not subject to the limitations of synthetic materials regarding composition and functionalization. However, in contrast to synthetic membranes with very well-defined micropores, such as microfabricated PDMS membranes, the porosity of biological membranes is not well-defined and may be a limitation for instance to block the migration of cells. Nevertheless, biological membranes present other advantages, such as a simple production process. These membranes can be stored for long periods of time (at least 2 weeks) without significant effects on stiffness. In summary, biological membranes provide the organs-on-chip and the tissue engineering communities with a powerful tool that can be tuned at will to modify its composition and mechanical properties to reproduce the in vivo ECM. Their integration into organ-on-a-chip devices has already started as reported by several groups. ${ }^{12-14,31}$ Given the new possibilities provided by such membranes, there is little doubt that they will increasingly replace synthetic ones in organs-on-chips in the years to come.

\section{ASSOCIATED CONTENT}

\section{(s) Supporting Information}

The Supporting Information is available free of charge at https://pubs.acs.org/doi/10.1021/acsbiomaterials.0c00515.

Figure S1, chip design; Figure S2, thickness of the vitrified and hydrogel-based biological membranes; Figure S3, representative image of a hydrogel CE membrane at rest and after exposure to a negative pressure of $-1.1 \mathrm{kPa}$; Figure $\mathrm{S} 4$, determination of the Young's modulus based on the pressure-deflection curve of the hydrogel CE membrane; Figure S5, impact of the storage of the vitrified $\mathrm{CE}$ membrane on its mechanical properties; Figure S6, impact of a two-week immersion in physiological medium on the mechanical properties of the vitrified CE membrane; Figure S7, force-indentation curves and force-volume images obtained by AFM; Figure S8, Young's modulus variance measured by AFM; Figure S9, membrane production, impact of the gelation temperature on the Young's modulus during the production of the hydrogel $\mathrm{CE}$ membrane (PDF)

\section{AUTHOR INFORMATION}

\section{Corresponding Author}

Olivier T. Guenat - Organs-on-Chip Technologies

Laboratory, ARTORG Center, University of Bern, Bern 3008, Switzerland; Department of Pulmonary Medicine and Department of General Thoracic Surgery, University Hospital of Bern, Bern 3008, Switzerland; Email: olivier.guenat@ artorg.unibe.ch

\section{Authors}

Pauline Zamprogno - Organs-on-Chip Technologies Laboratory, ARTORG Center, University of Bern, Bern 3008, Switzerland; 이이.org/0000-0003-1068-7304

Giuditta Thoma - Organs-on-Chip Technologies Laboratory, ARTORG Center, University of Bern, Bern 3008, Switzerland

Veronika Cencen - Laboratory for Bio- and NanoInstrumentation, Ecole Polytechnique Fédérale de Lausanne, Lausanne 1015, Switzerland

Dario Ferrari - Organs-on-Chip Technologies Laboratory, ARTORG Center, University of Bern, Bern 3008, Switzerland

Barbara Putz - Laboratory for Mechanics of Materials and Nanostructures, EMPA Swiss Federal Laboratories for Materials Science and Technology, Thun 3602, Switzerland

Johann Michler - Laboratory for Mechanics of Materials and Nanostructures, EMPA Swiss Federal Laboratories for Materials Science and Technology, Thun 3602, Switzerland

Georg E. Fantner - Laboratory for Bio- and NanoInstrumentation, Ecole Polytechnique Fédérale de Lausanne, Lausanne 1015, Switzerland

Complete contact information is available at: https://pubs.acs.org/10.1021/acsbiomaterials.0c00515

\section{Author Contributions}

O.T.G. and P.Z. designed the experiments. D.F. set up the bulge test assay. G.T. performed all the deflection measurements. V.C. performed AFM measurements. P.Z. carried out the cells experiments. P.Z., G.T., and O.T.G. analyzed and 
interpreted the data. P.Z. and O.T.G. wrote the manuscript. All authors reviewed the manuscript.

\section{Author Contributions}

${ }^{\dagger}$ P.Z. and G.T. contributed equally to this work.

\section{Notes}

The authors declare no competing financial interest.

\section{ACKNOWLEDGMENTS}

The authors thank the Swiss National Science Foundation for its financial support (project 185365) as well as the Microscopy Imaging Facility of the University of Bern (MIC) for its assistance. B.P. acknowledges funding from the EMPA POSTDOCS-II program, in the frame of the EUH2020 Marie Skłodowska-Curie grant (754364). G.E.F. acknowledges funding from H2020-UE Framework Programme for Research \& Innovation (2014-2020); ERC-2017CoG; InCell; Project 73091. V.C. acknowledges funding from the EU-H2020 Marie Skłodowska-Curie grant (754354).

\section{REFERENCES}

(1) Humphrey, J. D.; Dufresne, E. R.; Schwartz, M. A. Mechanotransduction and extracellular matrix homeostasis. Nat. Rev. Mol. Cell Biol. 2014, 15, 802-812.

(2) Yeh, Y.; Ling, J.; Chen, W.; Lin, H.; Tang, M. Mechanotransduction of matrix stiffness in regulation of focal adhesion size and number: reciprocal regulation of caveolin-1 and $\beta 1$ integrin. Sci. Rep. 2017, 7, 1-14.

(3) Balestrini, J. L.; Niklason, L. E. Extracellular Matrix as a Driver for Lung Regeneration. Ann. Biomed. Eng. 2015, 43, 568-576.

(4) Handorf, A. M.; Zhou, Y.; Halanski, M. A.; Li, W. J. Tissue stiffness dictates development, homeostasis, and disease progression. Organogenesis 2015, 11, 1-15.

(5) Huh, D.; Matthews, B. D.; Mammoto, A.; Montoya-Zavala, M.; Hsin, H. Y.; Ingber, D. E. Reconstituting Organ-Level Lung. Science (Washington, DC, U. S.) 2010, 328, 1662-1668.

(6) Stucki, J. D.; Hobi, N.; Galimov, A.; Stucki, A. O.; SchneiderDaum, N.; Lehr, C.-M.; Huwer, H.; Frick, M.; Funke-Chambour, M.; Geiser, T.; Guenat, O. T. Medium throughput breathing human primary cell alveolus-on- chip model. Sci. Rep. 2018, 8, 1-13.

(7) Lind, J. U.; Busbee, T. A.; Valentine, A. D.; Pasqualini, F. S.; Yuan, H.; Yadid, M.; Park, S. J.; Kotikian, A.; Nesmith, A. P.; Campbell, P. H.; Vlassak, J. J.; Lewis, J. A.; Parker, K. K. Instrumented cardiac microphysiological devices via multimaterial three-dimensional printing. Nat. Mater. 2017, 16, 303-308.

(8) Marsano, A.; Conficconi, C.; Lemme, M.; Occhetta, P.; Gaudiello, E.; Votta, E.; Cerino, G.; Redaelli, A.; Rasponi, M. Beating heart on a chip: A novel microfluidic platform to generate functional 3D cardiac microtissues. Lab Chip 2016, 16, 599-610.

(9) Tanyeri, M.; Tay, S. Viable cell culture in PDMS-based microfluidic devices. Methods Cell Biol. 2018, 148, 3-33.

(10) Toepke, M. W.; Beebe, D. J. PDMS absorption of small molecules and consequences in microfluidic applications. Lab Chip 2006, 6, 1484-1486.

(11) van Meer, B. J.; de Vries, H.; Firth, K. S.A.; van Weerd, J.; Tertoolen, L. G.J.; Karperien, H. B.J.; Jonkheijm, P.; Denning, C.; Ijzerman, A. P.; Mummery, C. L. Small molecule absorption by PDMS in the context of drug response bioassays. Biochem. Biophys. Res. Commun. 2017, 482, 323-328.

(12) Mondrinos, M. J.; Yi, Y. S.; Wu, N. K.; Ding, X.; Huh, D. Native extracellular matrix-derived semipermeable, optically transparent, and inexpensive membrane inserts for microfluidic cell culture. Lab Chip 2017, 17, 3146-3158.

(13) Zamprogno, P.; Wüthrich, S.; Achenbach, S.; Thoma, G.; Stucki, J. D.; Hobi, N.; Schneider-Daum, N.; Lehr, C.-M.; Huwer, H.; Geiser, T.; Schmid, R. A.; Guenat, O. T. Second - generation lung - on
- a - chip with an array of stretchable alveoli made with a biological membrane. Commun. Biol. 2021, 4, 168.

(14) Lee, J. S.; Romero, R.; Han, Y. M.; Kim, H. C.; Kim, C. J.; Hong, J.-S.; Huh, D. Placenta-on-a-chip: a novel platform to study the biology of the human placenta. J. Matern.-Fetal Neonat. Med. 2016, 29, 1046-1054.

(15) Wang, C.; Tanataweethum, N.; Karnik, S.; Bhushan, A. Novel Microfluidic Colon with an Extracellular Matrix Membrane. ACS Biomater. Sci. Eng. 2018, 4, 1377-1385.

(16) Zamprogno, P.; Wüthrich, S.; Achenbach, S.; Stucki, J. D.; Hobi, N.; Schneider-Daum, N.; Lehr, C.-M.; Huwer, H.; Geiser, T.; Schmid, R. A.; Guenat, O. T. Second-generation lung-on-a-chip array with a stretchable biological membrane. bioRxiv 2019, DOI: 10.1101/ 608919 .

(17) Dong, C.; Lv, Y. Application of collagen scaffold in tissue engineering: Recent advances and new perspectives. Polymers (Basel, Switz.) 2016, 8, 42 .

(18) Miranda-Nieves, D.; Chaikof, E. L. Collagen and Elastin Biomaterials for the Fabrication of Engineered Living Tissues. ACS Biomater. Sci. Eng. 2017, 3, 694-711.

(19) Krieg, M.; Fläschner, G.; Alsteens, D.; Gaub, B. M.; Roos, W. H.; Wuite, G. J. L.; Gaub, H. E.; Gerber, C.; Dufrãne, Y. F.; Müller, D. J. Atomic force microscopy-based mechanobiology. Nat. Rev. Phys. 2019, 1, 41-57.

(20) Nehls, S.; Janshoff, A. Elastic Properties of Pore-Spanning Apical Cell Membranes Derived from MDCK II Cells. Biophys. J. 2017, 113, 1822-1830.

(21) Gonçalves, R. P.; Agnus, G.; Sens, P.; Houssin, C.; Bartenlian, B.; Scheuring, S. Two-chamber AFM: Probing membrane proteins separating two aqueous compartments. Nat. Methods 2006, 3, 10071012.

(22) Sheng, J. Y.; Zhang, L. Y.; Li, B.; Wang, G. F.; Feng, X. Q. Bulge test method for measuring the hyperelastic parameters of soft membranes. Acta Mech. 2017, 228, 4187-4197.

(23) Kraft, O.; Volkert, C. A. Mechanical testing of thin films and small structures. Adv. Eng. Mater. 2001, 3, 99-110.

(24) Small, M. K.; Nix, W. D. Analysis of the accuracy of the bulge test in determining the mechanical properties of thin films. J. Mater. Res. 1992, 7, 1553-1563.

(25) Huang, C. K.; Lou, W. M.; Tsai, C. J.; Wu, T. C.; Lin, H. Y. Mechanical properties of polymer thin film measured by the bulge test. Thin Solid Films 2007, 515, 7222-7226.

(26) Gao, J.; Guo, D.; Santhanam, S.; Fedder, G. K. Material Characterization and Transfer of Large-Area Ultra-Thin Polydimethylsiloxane Membranes. J. Microelectromech. Syst. 2015, 24, 21702177.

(27) Odermatt, P. D.; Shivanandan, A.; Deschout, H.; Jankele, R.; Nievergelt, A. P.; Feletti, L.; Davidson, M. W.; Radenovic, A.; Fantner, G. E. High-Resolution Correlative Microscopy: Bridging the Gap between Single Molecule Localization Microscopy and Atomic Force Microscopy. Nano Lett. 2015, 15, 4896-4904.

(28) Frantz, C.; Stewart, K. M.; Weaver, V. M. The extracellular matrix at a glance. J. Cell Sci. 2010, 123, 4195-200.

(29) Burgstaller, G.; Oehrle, B.; Gerckens, M.; White, E. S.; Schiller, H. B.; Eickelberg, O. The instructive extracellular matrix of the lung: Basic composition and alterations in chronic lung disease. Eur. Respir. J. 2017, 50, 1601805.

(30) Dunphy, S. E.; Bratt, J. A. J.; Akram, K. M.; Forsyth, N. R.; El Haj, A. J. Hydrogels for lung tissue engineering: Biomechanical properties of thin collagen-elastin constructs. J. Mech. Behav. Biomed. Mater. 2014, 38, 251-259.

(31) Humayun, M.; Chow, C. W.; Young, E. W. K. Microfluidic lung airway-on-a-chip with arrayable suspended gels for studying epithelial and smooth muscle cell interactions. Lab Chip 2018, 18, 1298-1309.

(32) Discher, D. E. Tissue Cells Feel and Respond to the Stiffness of Their Substrate. Science (Washington, DC, U. S.) 2005, 310, 11391143.

(33) Chang, Y. R.; Raghunathan, V. K.; Garland, S. P.; Morgan, J. T.; Russell, P.; Murphy, C. J. Automated AFM force curve analysis for 
determining elastic modulus of biomaterials and biological samples. $J$. Mech. Behav. Biomed. Mater. 2014, 37, 209-218.

(34) McBane, J. E.; Vulesevic, B.; Padavan, D. T.; McEwan, K. A.; Korbutt, G. S.; Suuronen, E. J. Evaluation of a Collagen-Chitosan Hydrogel for Potential Use as a Pro-Angiogenic Site for Islet Transplantation. PLoS One 2013, 8, e77538.

(35) Ahearne, M.; Yang, Y.; Then, K. Y.; Liu, K. K. Non-destructive mechanical characterisation of UVA/riboflavin crosslinked collagen hydrogels. Br. J. Ophthalmol. 2008, 92, 268-271.

(36) Joshi, J.; Mahajan, G.; Kothapalli, C. R. Three-dimensional collagenous niche and azacytidine selectively promote time-dependent cardiomyogenesis from human bone marrow-derived MSC spheroids. Biotechnol. Bioeng. 2018, 115, 2013-2026.

(37) Elibol, K.; Bayer, B. C.; Hummel, S.; Kotakoski, J.; Argentero, G.; Meyer, J. C. Visualising the strain distribution in suspended twodimensional materials under local deformation. Sci. Rep. 2016, 6, 1-9.

(38) Elibol, K.; Hummel, S.; Bayer, B. C.; Meyer, J. C. New imaging modes for analyzing suspended ultra-thin membranes by double-tip scanning probe microscopy. Sci. Rep. 2020, 10, 1-14.

(39) Antoine, E. E.; Vlachos, P. P.; Rylander, M. N. Review of collagen i hydrogels for bioengineered tissue microenvironments: Characterization of mechanics, structure, and transport. Tissue Eng., Part B 2014, 20, 683-696.

(40) Holder, A. J.; Badiei, N.; Hawkins, K.; Wright, C.; Williams, P. R.; Curtis, D. J. Control of collagen gel mechanical properties through manipulation of gelation conditions near the sol-gel transition. Soft Matter 2018, 14, 574-580.

(41) Raub, C. B.; Suresh, V.; Krasieva, T.; Lyubovitsky, J.; Mih, J. D.; Putnam, A. J.; Tromberg, B. J.; George, S. C. Noninvasive assessment of collagen gel microstructure and mechanics using multiphoton microscopy. Biophys. J. 2007, 92, 2212-2222.

(42) Achilli, M.; Mantovani, D. Tailoring mechanical properties of collagen-based scaffolds for vascular tissue engineering: The effects of $\mathrm{pH}$, temperature and ionic strength on gelation. Polymers (Basel, Switz.) 2010, 2, 664-680.

(43) Leipzig, N. D.; Shoichet, M. S. The effect of substrate stiffness on adult neural stem cell behavior. Biomaterials 2009, 30, 6867-6878.

(44) Nawroth, J. C.; Barrile, R.; Conegliano, D.; van Riet, S.; Hiemstra, P. S.; Villenave, R. Stem cell-based Lung-on-Chips: The best of both worlds? Adv. Drug Delivery Rev. 2019, 140, 12-32.

(45) Engler, A. J.; Rehfeldt, F.; Sen, S.; Discher, D. E. Microtissue Elasticity: Measurements by Atomic Force Microscopy and Its Influence on Cell Differentiation. Methods Cell Biol. 2007, 83, 521545.

(46) Hinz, B. Mechanical aspects of lung fibrosis: A spotlight on the myofibroblast. Proc. Am. Thorac. Soc. 2012, 9, 137-147.

(47) Prager-Khoutorsky, M.; Lichtenstein, A.; Krishnan, R.; Rajendran, K.; Mayo, A.; Kam, Z.; Geiger, B.; Bershadsky, A. D. Fibroblast polarization is a matrix-rigidity-dependent process controlled by focal adhesion mechanosensing. Nat. Cell Biol. 2011, 13, 1457-1465.

(48) Mih, J. D.; Sharif, A. S.; Liu, F.; Marinkovic, A.; Symer, M. M.; Tschumperlin, D. J. A multiwell platform for studying stiffnessdependent cell biology. PLoS One 2011, 6, e19929.

(49) Shkumatov, A.; Thompson, M.; Choi, K. M.; Sicard, D.; Baek, K.; Kim, D. H.; Tschumperlin, D. J.; Prakash, Y. S.; Kong, H. Matrix stiffness-modulated proliferation and secretory function of the airway smooth muscle cells. Am. J. Physiol. - Lung Cell. Mol. Physiol. 2015, 308, L1125-L1135.

(50) Yeung, T.; Georges, P. C.; Flanagan, L. A.; Marg, B.; Ortiz, M.; Funaki, M.; Zahir, N.; Ming, W.; Weaver, V.; Janmey, P. A. Effects of substrate stiffness on cell morphology, cytoskeletal structure, and adhesion. Cell Motil. Cytoskeleton 2005, 60, 24-34.

(51) Plotnikov, S. V.; Pasapera, A. M.; Sabass, B.; Waterman, C. M. Force fluctuations within focal adhesions mediate ECM-rigidity sensing to guide directed cell migration. Cell 2012, 151, 1513-1527.

(52) Steward, A. J.; Kelly, D. J. Mechanical regulation of mesenchymal stem cell differentiation. J. Anat. 2015, 227, 717-731.
(53) Yang, C.; Tibbitt, M. W.; Basta, L.; Anseth, K. S. Mechanical memory and dosing influence stem cell fate. Nat. Mater. 2014, 13, $645-652$.

(54) Mih, J. D.; Marinkovic, A.; Liu, F.; Sharif, A. S.; Tschumperlin, D. J. Matrix stiffness reverses the effect of actomyosin tension on cell proliferation. J. Cell Sci. 2012, 125, 5974-5983. 